\title{
Deciphering the Complex Crystallography of a Strained Two-phase Alloy Using High-resolution STEM and Molecular Dynamics Calculations
}

\author{
Albina Borisevich, Eva Zarkadoula, Ying Yang and Easo George \\ Oak Ridge National Laboratory, Oak Ridge, Tennessee, United States
}

Ni-based superalloys are widely used in aircraft and power-generation turbines due to their exceptional mechanical properties, understood to be driven by the presence of the stable ordered L12-type precipitates $[1,2]$. Fe-Ni based alloys are considered as a lower-cost alternative, however, replicating the desired microstructure in those systems is difficult given that the precipitates are often metastable and can degrade [e.g. 3,4]. Recent computational and experimental work has demonstrated that, unlike the previously studied compositions, an alloy Fe-37.4Ni-6.1Al-2.9Ti (FNAT) can form stable precipitates that are rich in $\mathrm{Ti}$ and $\mathrm{Al}$ and achieve the desired improvements in mechanical properties [5]. Further studies have uncovered that even precipitate size can substantially alter the trajectory of the strain-driven deformation. In order to identify the underlying mechanism, both the detailed crystallography of the system and its dynamic behavior need to be explored.

In this work, we investigate the microstructure of the FNAT alloy that was thermally treated to produce 5-15 nm size L12 inclusions under a varying degree of strain using high resolution STEM. We examined three distinct strain states $(0 \%, 6.5 \%$, and $28.5 \%)$; the samples were prepared using FIB liftout. From the XRD data, only the $28.5 \%$ strained sample was determined to undergo martensitic transformation of the matrix. Fig. 1 shows the high-resolution images taken in [110] orientation of the precipitates for different strain states. All the samples demonstrate misorientation $\left(6-7^{\circ}\right)$ between the precipitates and the matrix (explored via diffraction and GPA analysis), resulting in an interesting contrast reversal in HAADF depending on which of the components is closer to the zone axis for the observation conditions; for example, Fig.1(a) shows L12 precipitate on-axis, while for Fig. 1(b), matrix is on-axis. For the $28.5 \%$ strained sample it was not possible to find matrix on-axis conditions due to large accumulated deformation and the onset of the fcc-bcc martensitic transition in the matrix mentioned above.

This orientational relationship was not observed in previous studies of the alloy with 20-50 nm precipitates, which showed complete coherence of $\mathrm{L}_{2}$ and the matrix before the onset of the martensitic deformation [5]. We have therefore initiated a molecular dynamics study to determine if the observed misorientation contributes to different deformation trajectory of the smaller-precipitate alloys. Another observation that can be made from Fig. 1 is that L12 particles keep their spherical shape even at the highest strain level. We plan to use molecular dynamics and dislocation analysis to study this behavior in detail.

Contrast reversal demonstrated in Fig. 1 makes the identification of precipitates ambiguous based on HAADF contrast, however EELS imaging has proven very useful in removing the ambiguity. Fig. 2 shows simultaneous HAADF image alongside an RGB composite of the $\mathrm{Fe}, \mathrm{Ni}$ and $\mathrm{Ti}$ atomic fractions derived from EELS. Comparing the two panels of Fig. 2 shows that HAADF contrast change can be completely absent for a smaller precipitate, even for the measured specimen thickness of $18 \mathrm{~nm}$. The prospects of using high-resolution imaging for collecting quantitative local structure information from strained alloys will also be discussed [6]. 


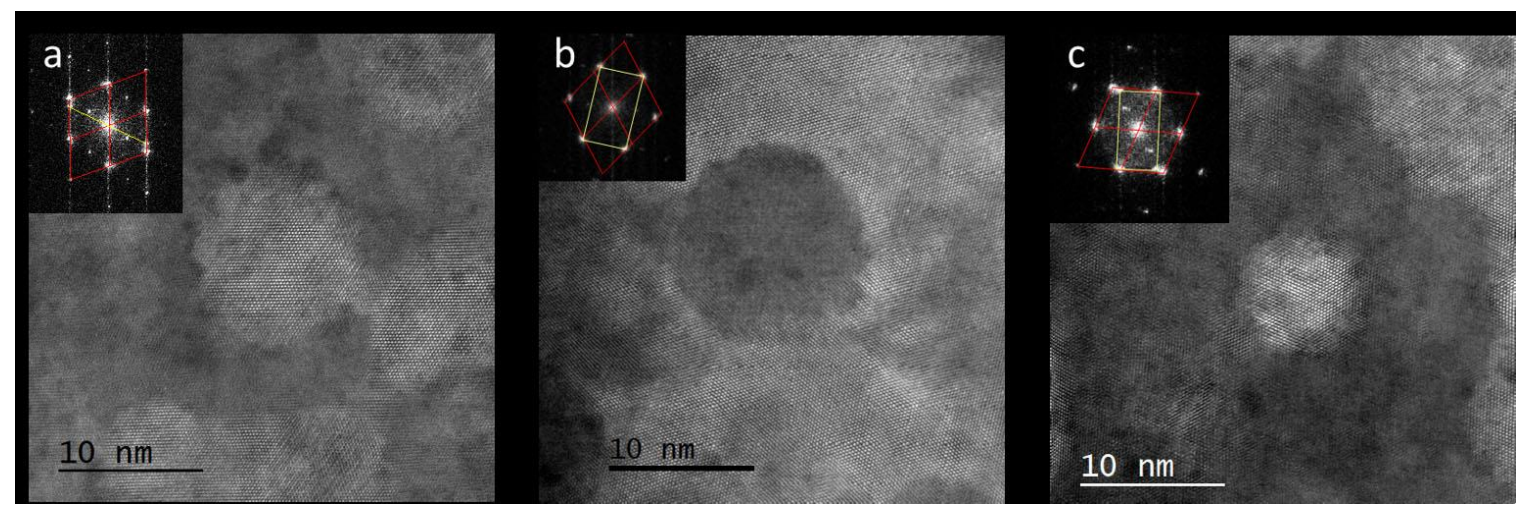

Figure 1. HAADF STEM images and corresponding diffractograms of FNAT medium entropy mixedphase alloys viewed down [110] axis showing orientational relationship of the L12 precipitates and the matrix for (a) unstrained case, (b) $6.5 \%$ tensile strain, (c) $28.5 \%$ tensile strain.
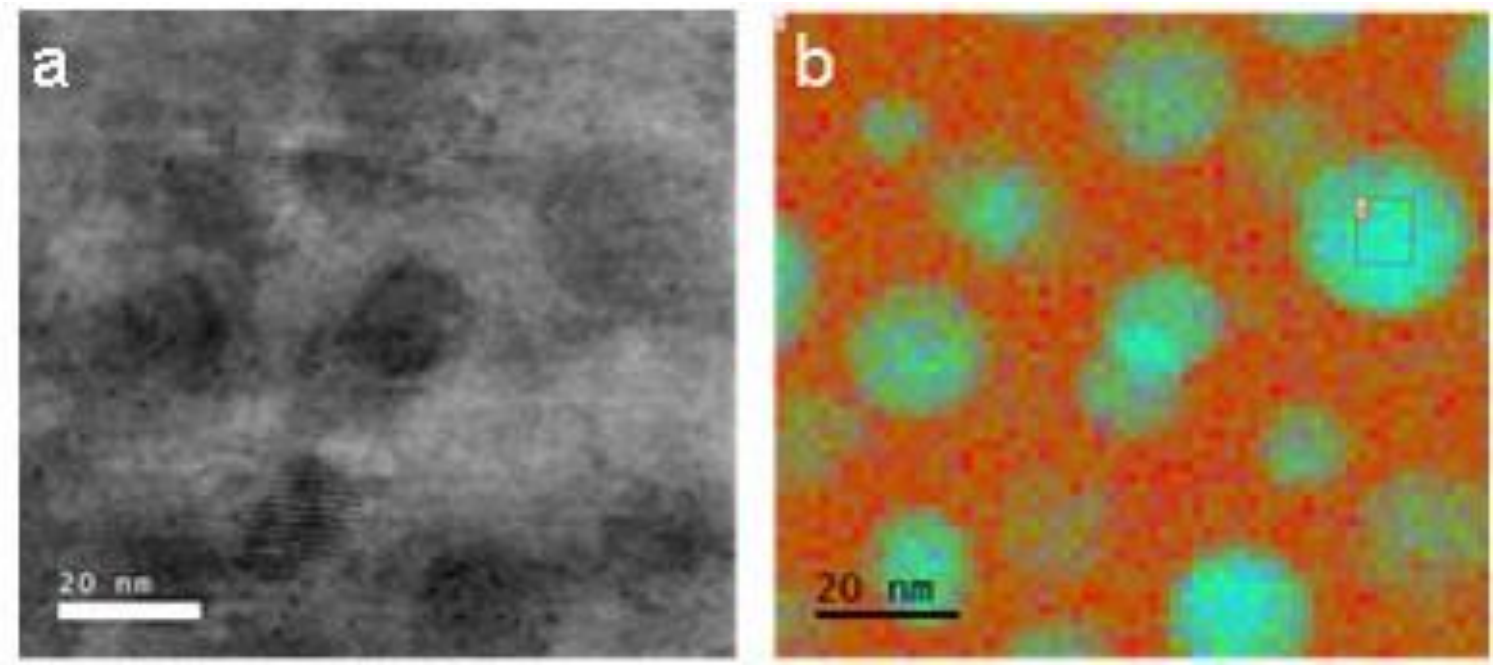

Figure 2. (a) HAADF STEM image of a strained FNAT alloy and (b) the corresponding RGB composite of the EELS compositional maps of Fe L (red), Ni L(green), and Ti L(blue) edges, demonstrating imperfect correspondence of the image contrast to the chemical signature of the precipitates.

\section{References}

[1] T.M. Pollock, S. Tin, J. Propuls. Power, 22361 (2006).

[2] M.J. Donachie, S.J. Donachie in Superalloys: a technical guide, ASM international (2002)

[3] A. Thompson, J. Brooks, Acta Metall., 302197 (1982).

[4] C.-M. Kuo, et al., Mater. Sci. Eng. A, 510289 (2009).

[5] Y. Yang et al., Materials \& Design in press, 108592 (2020)

[6] Research was sponsored by the Division of Materials Science and Engineering, Basic Energy

Sciences (BES), Office of Science, US Department of Energy (DOE). 